Kragujevac Journal of Mathematics

Volume 42(1) (2018), Pages 83-95.

\title{
NEW SUBCLASS OF MULTIVALENT HYPERGEOMETRIC MEROMORPHIC FUNCTIONS
}

\author{
M. ALBEHBAH ${ }^{1}$ AND M. DARUS ${ }^{2}$
}

\begin{abstract}
In this paper, we introduce a new class $\sum_{p}^{*}(A, B, k)_{a, c}$ for $-1 \leqslant$ $B<A \leqslant 1$ which consists of hypergeometric meromorphic functions of the form $L_{p}^{*}(a, c) f(z)=\frac{1}{z^{p}}+\sum_{n=0}^{\infty} \frac{(a)_{n+2}}{(c)_{n+2}} a_{n+p} z^{n+p}$ in $U^{*}=\{z: 0<|z|<1\}$. We determine sufficient conditions, distortion properties, radii of starlikeness and convexity and inclusion properties for the class $L_{p}^{*}(a, c) f(z)$.
\end{abstract}

\section{INTRODUCTION}

Let $\sum_{p}$ denote the class of meromorphic functions $f$ normalized by

$$
f(z)=\frac{1}{z^{p}}+\sum_{n=0}^{\infty} a_{n+p} z^{n+p},
$$

which are analytic and $p$-valent in the punctured unit disk $U^{*}=\{z: 0<|z|<1\}$.

For $0 \leq \alpha<p$, we denote by $\mathcal{S}_{p}^{*}(\alpha)$ and $\mathcal{K}_{p}(\alpha)$, the subclasses of $\sum_{p}$ consisting of all meromorphic functions which are, respectively, starlike of order $\alpha$ and convex of order $\alpha$ in $U^{*}$.

The classes $\mathcal{S}_{p}^{*}(\alpha), \mathcal{K}_{p}(\alpha)$ and various other subclasses of $\sum_{p}$ have been studied rather extensively by Aouf et al. [3-5], Ghanim and Darus [16], Srivastava [19], Kulkarni et al. [20], Morga [26,27], Owa et al. [28], Srivastava and Owa [29], Uralegaddi and Somantha [31,32], and Yang [33].

Key words and phrases. p-valent hypergeometric functions, meromorphic functions, starlike functions, convex functions, Hadamard product.

2010 Mathematics Subject Classification. Primary: 30C45. Secondary: 33C20, 30C85.

Received: January 17, 2016.

Accepted: October 12, 2016. 
For functions $f_{j},(j=1 ; 2)$ defined by

$$
f_{j}(z)=\frac{1}{z^{p}}+\sum_{n=0}^{\infty} a_{n+p, j} z^{n+p-1}
$$

we denote the Hadamard product (or convolution) of $f_{1}(z)$ and $f_{2}(z)$ by

$$
\left(f_{1} * f_{2}\right)(z)=\frac{1}{z^{p}}+\sum_{n=0}^{\infty} a_{n+p, 1} a_{n+p, 2} z^{n+p} .
$$

Let us define the function $\phi_{p}(a, c ; z)$ by

$$
\phi_{p}(a, c ; z)=\frac{1}{z^{p}}+\sum_{n=0}^{\infty}\left|\frac{(a)_{n+1}}{(c)_{n+1}}\right| a_{n} z^{n+p-1},
$$

for $c \neq 0,-1,-2, \ldots$ and $a \in C \backslash\{0\}, p \in N=1,2,3, \ldots$ where $(\lambda)_{n}$ is the Pochhammer symbol. We note that

$$
\phi(a, c ; z)=\frac{1}{z^{p}}{ }_{2} F_{1}(1, a, c ; z),
$$

where

$$
{ }_{2} F_{1}(1, a, c ; z)=\sum_{n=0}^{\infty} \frac{(1)_{n+1}(a)_{n+1}}{(c)_{n+1}} \frac{z^{n}}{n !},
$$

is the well-known Gaussian hypergeometric function. Corresponding to the function $\phi_{p}(a, c ; z)$, using the Hadamard product for $f \in \sum_{p}$, we define a new linear operator $L_{p}^{*}(a, c)$ on $\sum_{p}$ by

$$
L_{p}^{*}(a, c) f(z)=\phi(a, c ; z) * f(z)=\frac{1}{z^{p}}+\sum_{n=0}^{\infty}\left|\frac{(a)_{n+2}}{(c)_{n+2}}\right| a_{n+p} z^{n+p} .
$$

The meromorphic functions with the generalized hypergeometric functions were considered recently by Dziok and Srivastava [12,13], Liu [21], Liu and Srivastava [22-24], Cho and Kim [9], Ghanim and Darus [15].

For a function $f \in \sum_{p}$, Albehbah and Darus [2] introduced and studied the differential operator $I_{p}^{k} f(z)$

$$
I_{p}^{k} f(z)=\frac{1}{z^{p}}+\sum_{n=0}^{\infty} P(n, k) a_{n} z^{n}
$$

and by using (1.1) and (1.2), we define the operator $I^{k}\left(L_{p}^{*}(a, c) f(z)\right)$.

For a function $f \in \sum_{p}$, we define

$$
I^{0}\left(L_{p}^{*}(a, c) f(z)\right)=L_{p}^{*}(a, c) f(z),
$$


and for $k=1,2,3, \ldots$, we have

$$
\begin{aligned}
I^{k}\left(L_{p}^{*}(a, c) f(z)\right) & =z^{k}\left[\frac{1}{z^{k-1}}\left(I^{k-1} L_{p}^{*}(a, c) f(z)\right)\right]^{\prime}+\frac{(p+k)}{z^{p}} \\
& =\frac{1}{z^{p}}+\sum_{n=0}^{\infty} P(n+p, k)\left|\frac{(a)_{n+2}}{(c)_{n+2}}\right| a_{n+p} z^{n+p},
\end{aligned}
$$

where $P(n+p, k)=\frac{n+p !}{(n+p-k) !}$.

We note that $I^{k}\left(L_{1}^{*}(a, c) f\right)$ was studied earlier by Albehbah and Darus [1].

Also, it follows from (1.1) that (see [21])

$$
z\left(\left(L_{p}^{*}(a, c) f(z)\right)\right)^{\prime}=a L_{p}^{*}(a+1, c) f(z)-(a+p) L_{p}^{*}(a, c) .
$$

Now, let $-1 \leqslant B<A \leqslant 1$ and for all $z \in U^{*}$, a function $f \in \sum_{p}$ is said to be a member of the class $\sum_{p}^{*}(A, B, k)_{a, c}$ if it satisfies

$$
\left|\frac{z\left(I^{k} L^{*}(a, c) f(z)\right)^{\prime}+p I^{k} L^{*}(a, c) f(z)}{B z\left(I^{k} L^{*}(a, c) f(z)\right)^{\prime}+A p\left(I^{k} L^{*}(a, c) f(z)\right.}\right|<1 .
$$

The class $\sum_{1}^{*}(A, B, 0)_{a, c}=\sum^{*}(A, B)_{a, c}$ was studied by Morga [27].

Note that, for $a=c, p=1, \sum^{*}(1-2 \mu,-1, k)_{a, c}$ with $0 \leq \mu<1$, is the class introduced and studied in [1].

In Section 2, our first result concerns the coefficient estimates and distortion theorem for the class $\sum_{p}^{*}(A, B, k)_{a, c}$.

\section{Coefficient Estimates and Distortion Theorems}

Our first result provides a sufficient condition for a function $f$ analytic in $U^{*}$, to be in the class $\sum^{*}(A, B, k)_{a, c}$.

Theorem 2.1. Let the function $f$ be defined by (1.1). If

$$
\sum_{n=0}^{\infty} P(n+p, k)\left|\frac{(a)_{n+2}}{(c)_{n+2}}\right|[(n+p)(1-B)+p(1-A)]\left|a_{n+p}\right| \leqslant p(A-B),
$$

where $k \in N_{0}=N \cup\{0\},-1 \leq B<A \leq 1$, then $f \in \sum^{*}(A, B, k)_{a, c}$.

Proof. To prove Theorem 2.1, we show that if $f$ satisfies (2.1) then

$$
\begin{aligned}
M\left(f, f^{\prime}\right)= & \left|z\left(I^{k} L_{p}^{*}(a, c) f(z)\right)^{\prime}+p I^{k} L_{p}^{*}(a, c) f(z)\right| \\
& -\left|B z\left(I^{k} L_{p}^{*}(a, c) f(z)\right)^{\prime}+A p I^{k} L_{p}^{*}(a, c) f(z)\right| .
\end{aligned}
$$

Then for $0<|z|=r<1$ we have

$$
\begin{aligned}
M\left(f, f^{\prime}\right)= & \left|\sum_{n=0}^{\infty} P(n+p, k)\right| \frac{(a)_{n+2}}{(c)_{n+2}}\left|(n+2 p) a_{n+p} z^{n+p}\right| \\
& -\left|\frac{p(A-B)}{z^{p}}+\sum_{n=0}^{\infty} P(n+p, k)\right| \frac{(a)_{n+2}}{(c)_{n+2}}\left|((n+p) B+A p) a_{n+p} z^{n+p}\right| .
\end{aligned}
$$


This gives

$$
\begin{aligned}
r^{p} M\left(f, f^{\prime}\right) \leq & \sum_{n=0}^{\infty} P(n+p, k)\left|\frac{(a)_{n+2}}{(c)_{n+2}}\right|[(n+p)(1-B)+p(1-A)]\left|a_{n+p}\right| r^{n+2 p} \\
& -p(A-B) .
\end{aligned}
$$

The inequality in $(2.2)$ holds true for all $r(0<r<1)$. Therefore, by letting $r \longrightarrow 1$ in (2.2), we have

$$
\begin{aligned}
M\left(f, f^{\prime}\right) & \leq \sum_{n=0}^{\infty} P(n+p, k)\left|\frac{(a)_{n+2}}{(c)_{n+2}}\right|[(n+p)(1-B)+p(1-A)]\left|a_{n+p}\right|-p(A-B) \\
& \leq 0
\end{aligned}
$$

by the hypothesis (2.1). Hence it follows that

$$
\left|z\left(I^{k} L_{p}^{*}(a, c) f(z)\right)^{\prime}+p I^{k} L_{p}^{*}(a, c) f(z)\right| \leq\left|B z\left(I^{k} L_{p}^{*}(a, c) f(z)\right)^{\prime}+A p I^{k} L_{p}^{*}(a, c) f(z)\right|,
$$

so that $f \in \sum_{p}^{*}(A, B, k)_{a, c}$.

Our assertion in Theorem 2.1 is sharp for function $f$ of the form:

$$
f_{n+p}(z)=\frac{1}{z^{p}}+\frac{\left|(c)_{n+2}\right| p(A-B)}{\left|(a)_{n+2}\right| P(n+p, k)((n+p)(1-B)+p(1-A))} z^{n},
$$

where $n \geq 0 k \in N_{0},-1 \leq B<A \leq 1$.

Corollary 2.1. Let the function $f$ be defined by (1.1) and let $f \in \sum_{p}$. If $f \in$ $\sum_{p}^{*}(A, B, k)_{a, c}$. Then

$$
\left|a_{n+p}\right| \leq \frac{p(A-B)\left|(c)_{n+2}\right|}{P(n+p, k)[(n+p)(1-B)+p(1-A)]\left|(a)_{n+2}\right|},
$$

where $n \geq 0, k \in N_{0},-1 \leq B<A \leq 1$.

The result (2.4) is sharp for function $f_{n+p}$ given by (2.3).

Corollary 2.2. For $k=0, A=1$, and $B=-1$ in Theorem 2.1, we have

$$
\left|\sum_{n=0}^{\infty} \frac{(a)_{n+2}}{(c)_{n+2}}\right|(n+p)\left|a_{n}+p\right| \leq 1
$$

and therefore the function $L_{p}^{*}(a, c) f$ is starlike in $U^{*}$.

Corollary 2.3. For $k=1, A=1$, and $B=-1$ in Theorem 2.1, we have

$$
\left|\sum_{n=0}^{\infty} \frac{(a)_{n+2}}{(c)_{n+2}}\right|(n+p)^{2}\left|a_{n}+p\right| \leq 1
$$

and therefore the function $L_{p}^{*}(a, c) f$ is convex in $U^{*}$.

The growth and distortion properties for function $f$ of the class $\sum_{p}^{*}(A, B, k)_{a, c}$ are given in the following result. 
Theorem 2.2. If the function $f$ be defined by (1.1) is in the class $\sum_{p}^{*}(A, B, k)_{a, c}$, then for $|z|=r, 0<r<1$, we have

$$
\frac{1}{r^{p}}-\frac{p(A-B)}{[2 p-p(A+B)]} r^{p} \leq|f(z)| \leq \frac{1}{r^{p}}+\frac{p(A-B)}{[2 p-p(A+B)]} r^{p}
$$

and

$$
\frac{p}{r^{p+1}}-\frac{p(A-B)}{[2 p-p(A+B)]} r^{p-1} \leq\left|f^{\prime}(z)\right| \leq \frac{p}{r^{p+1}}+\frac{p(A-B)}{[2 p-p(A+B)]} r^{p-1}
$$

with equality for

$$
f(z)=\frac{1}{z^{p}}+\frac{p(A-B)}{[2 p-p(A+B)]} z^{p} .
$$

Proof. Since $f \in \sum_{p}^{*}(A, B, k)_{a, c}$, Theorem 2.1 readily yields the inequality

$$
\sum_{n=0}^{\infty}\left|\frac{(c)_{n+2}}{(a)_{n+2}}\right| a_{n+p} \leq \frac{p(A-B)}{P(p, k)[2 p-p(A+B)]} .
$$

Thus, for $0<|z|=r<1$, and making use of (2.5) we have

$$
\begin{gathered}
|f(z)| \leq \frac{1}{|z|^{p}}+\sum_{n=0}^{\infty}\left|\frac{(c)_{n+2}}{(a)_{n+2}}\right|\left|a_{n+p}\right||z|^{n+p} \\
\leq \frac{1}{r^{p}}+r^{p} \sum_{n=0}^{\infty}\left|\frac{(c)_{n+2}}{(a)_{n+2}}\right|\left|a_{n+p}\right| \leq \frac{1}{r^{p}}+\frac{p(A-B)}{[2 p-p(A+B)]} r^{p}
\end{gathered}
$$

and

$$
\begin{gathered}
|f(z)| \geq \frac{1}{|z|^{p}}-\sum_{n=0}^{\infty}\left|\frac{(c)_{n+2}}{(a)_{n+2}}\right|\left|a_{n+p}\right||z|^{n+p} \\
\geq \frac{1}{r^{p}}-r^{p} \sum_{n=0}^{\infty}\left|\frac{(c)_{n+2}}{(a)_{n+2}}\right|\left|a_{n+p}\right| \geq \frac{1}{r^{p}}-\frac{p(A-B)}{[2 p-p(A+B)]} r^{p} .
\end{gathered}
$$

Also from Theorem 2.1, it follows that

$$
\begin{aligned}
\sum_{n=0}^{\infty}\left|\frac{(c)_{n+2}}{(a)_{n+2}}\right|(n+p)\left|a_{n+p}\right| & \leq \frac{p(A-B)}{P(p-1, k)[2 p-p(A+B)]} \\
\left|f^{\prime}(z)\right| & \leq \frac{p}{|z|^{p+1}}+\sum_{n=0}^{\infty}\left|\frac{(c)_{n+2}}{(a)_{n+2}}\right|(n+p)\left|a_{n+p}\right||z|^{n+p-1} \\
& \leq \frac{p}{r^{p+1}}+r^{p-1} \sum_{n=0}^{\infty}\left|\frac{(c)_{n+2}}{(a)_{n+2}}\right|(n+p)\left|a_{n+p}\right| \\
& \leq \frac{p}{r^{p+1}}+\frac{p(A-B)}{[2 p-p(A+B)]} r^{p-1}
\end{aligned}
$$


and

$$
\begin{aligned}
\left|f^{\prime}(z)\right| & \geq \frac{p}{|z|^{p+1}}+\sum_{n=0}^{\infty}\left|\frac{(c)_{n+2}}{(a)_{n+2}}\right|(n+p)\left|a_{n+p}\right||z|^{n+p-1} \\
& \geq \frac{p}{r^{p+1}}+r^{p-1} \sum_{n=0}^{\infty}\left|\frac{(c)_{n+2}}{(a)_{n+2}}\right|(n+p)\left|a_{n+p}\right| \\
& \geq \frac{p}{r^{p+1}}+\frac{p(A-B)}{[2 p-p(A+B)]} r^{p-1} .
\end{aligned}
$$

Thus, the proof of the theorem is complete.

\section{RAdi of Starlikeness And CONVEXity}

The radii of starlikeness and convexity for the class $\sum_{p}^{*}(A, B, k)_{a, c}$ is given by the following theorems.

Theorem 3.1. If the function $f$ defined by (1.1) is in the class $\sum_{p}^{*}(A, B, k)_{a, c}$, then $f$ is meromorphically starlike of order $\delta(0 \leq \delta \leq 1)$ in the disk $|z|<r_{1}$ where

$$
\begin{aligned}
r_{1} & =r_{1}(A, B, k, \delta) \\
& =\inf _{n \geq_{0}}\left\{\frac{P(n+p, k)(p-\delta)[(n+p)(1-B)+p(1-A)]}{p(A-B)(n+3 p-\delta)}\right\}^{\frac{1}{n+2 p}},
\end{aligned}
$$

where the result is sharp for the functions $f_{n}$ given by (2.3).

Proof. It suffices to prove that

$$
\left|\frac{z\left(L_{p}^{*}(a, c) f(z)\right)^{\prime}}{L_{p}^{*}(a, c) f(z)}+p\right| \leq p-\delta
$$

for $|z|<r_{1}$ we have

$$
\begin{aligned}
\left|\frac{z\left(L_{p}^{*}(a, c) f(z)\right)^{\prime}}{L_{p}^{*}(a, c) f(z)}+p\right| & =\left|\frac{\sum_{n=0}^{\infty}\left|\frac{(c)_{n+2}}{(a)_{n+2}}\right|(n+2 p) a_{n+p} z^{n+p}}{\frac{1}{z^{p}}+\sum_{n=0}^{\infty} \frac{(c)_{n+2}}{(a)_{n+2}} a_{n+p} z^{n+p}}\right| \\
& \leq \frac{\sum_{n=0}^{\infty}\left|\frac{(c)_{n+2}}{(a)_{n+2}}\right|(n+2 p)\left|a_{n+p}\right||z|^{n+2 p}}{1-\sum_{n=0}^{\infty}\left|\frac{(a)_{n+2}}{(c)_{n+2}}\right|\left|a_{n+p}\right||z|^{n+2 p}}
\end{aligned}
$$

Hence (3.1) holds true if

$$
\sum_{n=0}^{\infty}\left|\frac{(c)_{n+2}}{(a)_{n+2}}\right|(n+2 p)\left|a_{n+p}\right||z|^{n+2 p} \leqslant(p-\delta)\left(1-\sum_{n=0}^{\infty}\left|\frac{(a)_{n+2}}{(c)_{n+2}}\right|\left|a_{n+p}\right||z|^{n+2 p}\right)
$$

or

$$
\sum_{n=0}^{\infty}\left|\frac{(a)_{n+2}}{(c)_{n+2}}\right| \frac{(n+3 p-\delta)}{(p-\delta)}\left|a_{n+p}\right||z|^{n+2 p} \leqslant 1
$$


With the aid of (2.1) and (3.2), it is true if

$$
\left|\frac{(a)_{n+2}}{(c)_{n+2}}\right| \frac{(n+3 p-\delta)}{(p-\delta)}|z|^{n+2 p} \leqslant \frac{P(n, k)[(n+p)(1-B)+p(1-A)]\left|(a)_{n+2}\right|}{p(A-B)\left|(c)_{n+2}\right|},
$$

where $n \geq 0$. Solving (3.3) with respect to $|z|$, we obtain

$$
|z|<\left\{\frac{P(n+p, k)(p-\delta)[(n+p)(1-B)+p(1-A)]}{p(A-B)(n+3 p-\delta)}\right\}^{\frac{1}{n+2 p}}
$$

and this completes the proof of Theorem 3.1.

Theorem 3.2. If the function $f$ defined by (1.1) is in the class $\sum_{p}^{*}(A, B, k)_{a, c}$, then $f$ is meromorphically convex of order $\delta(0 \leq \delta \leq 1)$ in the disk $|z|<r_{2}$ where

$$
\begin{aligned}
r_{2} & =r_{2}(A, B, k, \delta) \\
& =\inf _{n \geq_{0}}\left\{\frac{P(n+p-1, k)(p-\delta)[(n+p)(1-B)+p(1-A)]}{p(A-B)(n+3 p-\delta)}\right\}^{\frac{1}{n+2 p}},
\end{aligned}
$$

where the result is sharp for the functions $f_{n}$ given by (2.3).

Proof. By using the technique employed in the proof of Theorem 3.1, and with the aid of Theorem 2.1, we can show that

$$
\left|\frac{z\left(L_{p}^{*}(a, c) f(z)\right)^{\prime \prime}}{L_{p}^{*}(a, c) f(z)}+p+1\right| \leq p-\delta
$$

for $|z|<r_{2}$. Thus we have the assertion of Theorem 3.2.

\section{Convex Linear Combination}

Our next result involves a linear combination of several function of the type (2.3).

Theorem 4.1. Let

$$
f_{p-1}(z)=\frac{1}{z^{p}}
$$

and

$$
f_{n+p}(z)=\frac{1}{z^{p}}+\frac{p(A-B)\left|(c)_{n+2}\right|}{P(n+p, k)[(n+p)(1-B)+p(1-A)]\left|(a)_{n+2}\right|} z^{n+p},
$$

for $n \geq 0$ and $k \in N_{0}$.

Then $f \in \sum_{p}^{*}(A, B, k)_{a, c}$ if and only if it can expressed in the form

$$
f(z)=\sum_{n=0}^{\infty} \lambda_{p+n-1} f_{p+n-1}(z)
$$

where $\lambda_{p+n-1} \geq 0$ and $\sum_{n=0}^{\infty} \lambda_{p+n-1}=1$. 
Proof. From (4.1), (4.2) and (4.3), it is easily seen that

$$
\begin{aligned}
f(z) & =\sum_{n=0}^{\infty} \lambda_{p+n-1} f_{p+n-1}(z) \\
& =\frac{1}{z^{p}}+\sum_{n=0}^{\infty} \frac{p(A-B)\left|(c)_{n+2}\right| \lambda_{n+p}}{P(n+p, k)[(n+p)(1-B)+p(1-A)]\left|(a)_{n+1}\right|} z^{n+p} \\
& =: \frac{1}{z^{p}}+\sum_{n=0}^{\infty} b_{n+p} z^{n+p} .
\end{aligned}
$$

Since

$$
\begin{aligned}
& \sum_{n=0}^{\infty} \frac{P(n+p, k)[(n+p)(1-B)+p(1-B)]\left|(a)_{n+2}\right|}{p(A-B)\left|(c)_{n+2}\right|} \lambda_{n+p} \\
& \times \frac{p(A-B)\left|(c)_{n+2}\right|}{P(n+p, k)[(n+p)(1-B)+p(1-A)]\left|(a)_{n+1}\right|} \\
= & \sum_{n=0}^{\infty} \lambda_{n+p}=1-\lambda_{p-1} \leq 0,
\end{aligned}
$$

it follows from Theorem 2.1 that the function $f \in \sum_{p}^{*}(A, B, k)_{a, c}$. Conversely, let us suppose that $f \in \sum_{p}^{*}(A, B, k)_{a, c}$. Since

$$
\left|a_{n+p}\right| \leq \frac{p(A-B)\left|(c)_{n+2}\right|}{P(n+p, k)[(n+p)(1-B)+p(1-A)]\left|(a)_{n+1}\right|}, \quad n \geq 0, k \in N_{0} .
$$

Setting

$$
\lambda_{n+p}=\frac{P(n+p, k)[(n+p)(1-B)+p(1-B)]\left|(a)_{n+2}\right|}{p(A-B)\left|(c)_{n+2}\right|}\left|a_{n+p}\right|,
$$

where $n \geq 0, k \in N_{0}, p \in N$ and

$$
\lambda_{p-1}=1-\sum_{n=0}^{\infty} \lambda_{n+p}
$$

it follows that $f(z)=\sum_{n=0}^{\infty} \lambda_{p+n-1} f_{p+n-1}(z)$.

Theorem 4.2. The class $\sum_{p}^{*}(A, B, k)_{a, c}$ is closed under convex linear combination.

Proof. Suppose that the function $f_{1}$ and $f_{2}$ defined by

$$
f_{j}(z)=\frac{1}{z^{p}}+\sum_{n=0}^{\infty}\left|a_{n+p j}\right| z^{n+p}, \quad j=1,2 ; z \in U^{*},
$$

are in the class $\sum_{p}^{*}(A, B, k)_{a, c}$.

Setting

$$
f(z)=\mu f_{1}(z)+(1-\mu) f_{2}(z), \quad 0 \leq \mu \leq 1
$$


we find from (4.4) that

$$
f(z)=\frac{1}{z^{p}}+\sum_{n=0}^{\infty}\left|\frac{(a)_{n+2}}{(c)_{n+2}}\right|\left\{\left|\mu a_{n+p, 1}+(1-\mu) a_{n+p, 2}\right|\right\} z^{n+p}, \quad 0 \leqslant \mu \leqslant 1, p \in N, z \in U^{*} .
$$

In view of Theorem 2.1, we have

$$
\begin{aligned}
& \quad \sum_{n=0}^{\infty}(P(n+p, k)[(n+p)(1-B)+p(1-A)])\left|\frac{(a)_{n+2}}{(c)_{n+2}}\right|\left\{\left|\mu a_{n+p, 1}+(1-\mu) a_{n+p, 2}\right|\right\} \\
& \quad \times \mu \sum_{n=0}^{\infty}(P(n+p, k)[(n+p)(1-B)+p(1-A)])\left|\frac{(a)_{n+2}}{(c)_{n+2}}\right|\left|a_{n+p, 1}\right| \\
& \quad+(1-\mu) \sum_{n=0}^{\infty}(P(n+p, k)[(n+p)(1-B)+p(1-A)])\left|\frac{(a)_{n+2}}{(c)_{n+2}}\right|\left|a_{n+p, 2}\right| \\
& \leq \mu p(A-B)+(1-\mu) p(A-B)=p(A-B),
\end{aligned}
$$

which shows that $f \in \sum_{p}^{*}(A, B, k)_{a, c}$. Hence the theorem is complete.

\section{InClusion Properties}

We begin by recalling the following result (popularly known as Jack's Lemma), which we shall apply in proving our inclusion theorem (Theorem 5.1 below).

Lemma 5.1 (Jack [18]). Let the (nonconstant) function $\omega(z)$ be analytic in $U^{*}$ with $\omega(0)=0$. If $|\omega(z)|$ attains its maximum value on the circle $|z|=r<1$ at a point $z_{0} \in U^{*}$, then

$$
z_{0} \omega^{\prime}\left(z_{0}\right)=\gamma \omega\left(z_{0}\right)
$$

where $\gamma$ is a real number and $\gamma \geq 1$.

Miller and Mocanu [25] gave proof to this lemma with the additional statement and made extensive use of it in their work on differential subordinations.

Theorem 5.1. If

$$
a \geq \frac{p(A-B)}{B+1}, \quad-1<B<A \leq 1, p \in N
$$

then

$$
\sum_{p}^{*}(A, B, k)_{a+1, c} \subset \sum_{p}^{*}(A, B, k)_{a, c}
$$

Proof. Let $f \in \sum_{p}^{*}(A, B, k)_{a+1, c}$ and suppose that

$$
\frac{z\left(I^{k}\left(L_{p}^{*}(a, c) f(z)\right)\right)^{\prime}}{\left(I^{k} L_{p}^{*}(a, c) f(z)\right)}=-p \frac{1+A \omega(z)}{1+B \omega(z)}
$$


where the function is either analytic or meromorphic in $U^{*}$, with $\omega(0)=0$. Then, by using (1.4) and (5.1), we have

$$
a \frac{\left(I^{k}\left(L_{p}^{*}(a+1, c) f(z)\right)\right)}{\left(I^{k} L_{p}^{*}(a, c) f(z)\right)}=\frac{a+[a B-p(A-B)] \omega(z)}{1+B \omega(z)} .
$$

Upon differentiating both sides of (5.2) with respect to $z$ logarithmically, if we make use of (1.4) once again, we obtain

$$
\begin{aligned}
& \frac{z\left(I^{k}\left(L_{p}^{*}(a+1, c) f(z)\right)\right)^{\prime}}{\left(I^{k} L_{p}^{*}(a+1, c) f(z)\right)} \\
= & -p \frac{1+A \omega(z)}{1+B \omega(z)}-\frac{p(A-B) z \omega^{\prime}(z)}{[1+B \omega(z)]\{a+[a B-p(A-B)] \omega(z)\}} .
\end{aligned}
$$

If we suppose now that

$$
\max _{|z| \leqslant\left|z_{0}\right|}|\omega(z)|=\left|\omega\left(z_{0}\right)\right|=1, \quad z_{0} \in U^{*}
$$

and apply Jack's Lemma, we find that

$$
z_{0} \omega^{\prime}\left(z_{0}\right)=\gamma \omega\left(z_{0}\right), \quad \gamma \geq 1
$$

Writing $w\left(z_{0}\right)=e^{i \theta}(0 \leq \theta<2 \pi)$ and setting $z=z_{0}$ in (5.3), we get

$$
\begin{aligned}
& \left|\frac{z_{0}\left(I^{k} L^{*}(a+1, c) f(z)\right)^{\prime}+p I^{k} L^{*}(a+1, c) f\left(z_{0}\right)}{B z_{0}\left(I^{k} L^{*}(a+1, c) f\left(z_{0}\right)\right)^{\prime}+A p\left(I^{k} L^{*}(a+1, c) f\left(z_{0}\right)\right.}\right|^{2}-1 \\
= & \left|\frac{(a+\gamma)+[a B-p(A-B)] e^{i \theta}}{a+[a B-\gamma-p(A-B)] e^{i \theta}}\right|^{2}-1 \\
= & \frac{2 \gamma(1+\cos \theta)[a(B+1)-p(A-B)]}{\left|a+[a B-\gamma-p(A-B)] e^{i \theta}\right|} \\
\geq & 0
\end{aligned}
$$

where $a \geqslant \frac{p(A-B)}{B+1},-1<B<A \leq 1, p \in N$, which obviously contradicts our hypothesis that

$$
f \in \sum_{p}^{*}(A, B, k)_{a+1, c}
$$

Thus we must have

$$
|\omega(z)|<1, \quad z \in U
$$

and so, from (5.1), we conclude that $f \in \sum_{p}^{*}(A, B, k)_{a, c}$, which evidently completes the proof of Theorem 5.1. 


\section{REMARKS}

Hypergeometric functions are of special interests among the complex analysts, and their main interest are on the properties and characterizations. The work has been around as early as in the 1900s. The classical results can be found in the books written by Exton [14], Bailey [6] and many other articles by many different authors, for example: Carlson and Shaffer [8] and the recent one by Ghany [17]. Too many researchers have studied the geometrical properties of this class and discovered many results. This type of functions is then convolute with the meromorphic type of functions to form a set of new class of functions. We mentioned before, the meromorphic functions with the generalized hypergeometric functions have been considered by many such as by Dziok and Srivastava [12, 13], Liu [21], Liu and Srivastava [22-24], Cho and Kim [9], and others (see $[7,10,11,30]$ ). Motivated by their results, we defined the generalized hypergeometric functions with the differential operator of meromorphic type, and several interesting results are obtained. Other applications such as the Fekete-Szegö problems, Hankel determinant and other coefficient related problems are yet to be considered for this new class.

Acknowledgements. This work was supported by MOHE: FRGS/1/2016/STG06/ $\mathrm{UKM} / 01 / 1$.

\section{REFERENCES}

[1] M. Albehbah and M. Darus, On new subclasses of meromorphic univalent functions with positive coefficients, Far East J. Math. Sci. 97 (4) (2015), 481-493.

[2] M. Albehbah and M. Darus, Subclasses of meromorphic multivalent functions, Acta Univ. Apul. Math. Inform. 43 (2015), 157-167.

[3] M. K. Aouf, New criteria for multivalent meromorphic starlike functions of order $\alpha$, Proc. Japan. Acad. Ser. A. Math. Sci. 69 (1993), 66-70.

[4] M. K. Aouf and H. M. Hossen, New criteria for meromorphic p-valent starlike functions, Tsukuba J. Math. 8 (17) (1993), 481-486.

[5] M. K. Aouf and H. M. Srivastava, A new criterion for meromorphically p-valent convex functions of order alpha, Math. Sci. Res. Hot-Line 8 (2) (1997), 7-12.

[6] W. N. Bailey, Basic hypergeometric series, Ch. 8 in Generalised Hypergeometric Series, Cambridge Univ. Press, pp. 65-72, 1935.

[7] S. K. Bajpai, A note on a class of meromorphic univalent functions, Rev. Roumaine Math. Pures Appl. 22 (3) (1977), 295-297.

[8] B. C. Carlson and D. B. Shaffer, Starlike and prestarlike hypergeometric functions, SIAM J. Math. Anal. 15 (1984), 737-745.

[9] N. E. Cho and I. H. Kim, Inclusion properties of certain classes of meromorphic functions associated with the generalized hypergeometric function, Appl. Math. Comput. 187 (2007), 115121.

[10] N. E. Cho, S. H. Lee and S. Owa, A class of meromorphic univalent functions with positive coefficients, Kobe J. Math. 4 (1) (1987), 43-50.

[11] Y. Dinggong, On a classes of meromorphic starlike multivalent functions, Bull. Inst. Math. Acad. Sin. 24 (1996), 151-157. 
[12] J. Dziok and H. M. Srivastava, Certain subclasses of analytic functions associated with the generalized hypergeometric function, Integral Transforms Spec. Funct. 14 (1) (2003), 7-18.

[13] J. Dziok and H. M. Srivastava, Some subclasses of analytic functions with fixed argument of coefficients associated with the generalized hypergeometric function, Adv. Stud. in Contemp. Math. 5 (2) (2002), 115-125.

[14] H. Exton, q-Hypergeometric Functions and Applications, John Willy \& Sons, 1983.

[15] F. Ghanim and M. Darus, New subclass of multivalent hypergeometric meromorphic functions, Int. J. Pure Appl. Math. 61 (3) (2010), 269-280.

[16] F. Ghanim, M. Darus and S. Sivasubramanian, New subclass of hypergeometric meromorphic functions, Far East J. Math. Sci. 34 (2) (2009), 245-256.

[17] H. A. Ghany, q-Derivative of basic hypergeometric series with respect to parameters, Int. J. Math. Anal. 3 (33) (2009), 1617-1632.

[18] I. S. Jack, Functions starlike and convex of order $\alpha$, J. Lond. Math. Soc, 2 (3) (1971), 469-474.

[19] S. B. Joshi and H. M. Srivastava, A certain family of meromorphically multivalent functions, Comput. Math. Appl. 38 (3-4)(1999), 201-211.

[20] S. R. Kulkarni, U. H. Naik and H. M. Srivastava, A certain class of meromorphically p-valent quasi-convex functions, Pan Amer. Math. J. 8 (1) (1998), 57-64.

[21] J. L. Liu, A linear operator and its applications on meromorphic p-valent functions, Bull. Inst. Math. Acad. Sin. 31 (1) (2003), 23-32.

[22] J. L. Liu and H. M. Srivastava, A linear operator and associated families of meromorphically multivalent functions, J. Math. Anal. Appl. 259 (2001), 566-581.

[23] J. L. Liu and H. M. Srivastava, Certain properties of the Dziok-Srivastava operator, Appl. Math. Comput. 159 (2) (2004), 485-493.

[24] J. L. Liu and H. M. Srivastava, Classes of meromorphically multivalent functions associated with the generalized hypergeometric function, Math. Comput. Modell. 39 (2004), 21-34.

[25] S. S. Miller and P. T. Mocanu, Second order differential inequalities in the complex plane, J. Math. Anal. Appl. 65 (1978), 289-305.

[26] M. L. Mogra, Meromorphic multivalent functions with positive coefficients I, Math. Japon. 35 (1990), 1-11.

[27] M. L. Mogra, Meromorphic multivalent functions with positive coefficients II, Math. Japon. 35 (1990), 1089-1098.

[28] S. Owa, H. E. Darwish and M. K. Aouf, Meromorphic multivalent functions with positive and fixed second coefficients, Math. Japan. 46 (1997), 231-236.

[29] H. M. Srivastava and S. Owa, Editors, Current Topics in Analytic Function Theory, World Sci. Sing., 1992.

[30] B. A. Uralegaddi and M. D. Ganigi, A certain class of meromorphically starlike functions with positive coefficients, Pure Appl. Math. Sci. 26 (1987), 75-80.

[31] B. A. Uralegaddi and C. Somanatha, Certain classes of meromorphic multivalent functions, Tamkang J. Math. 23 (1992), 223-231.

[32] B. A. Uralegaddi and C. Somanatha, New criteria for meromorphic starlike univalent functions, Bull. Aust. Math. Soc. 43 (1991), 137-140.

[33] D. G. Yang, On new subclasses of meromorphic p-valent functions, J. Math. Res. Exp. 15 (1995), $7-13$. 
${ }^{1}$ School of Mathematical Sciences

Faculty of Science and Technology

Universiti KebangsaAn Malaysia

BAngi 43600, SElangor D. EhSAn, MAlaysia

E-mail address: malbehbah@yahoo.com

School of Mathematical Sciences

Faculty of Science And TeChnology

Universiti Kebangsaan Malaysia

Bangi 43600, Selangor D. Ehsan, Malaysia

E-mail address: maslina@ukm.edu.my 